\title{
The future of personalized medicine
}

\section{Interview with Dr Richard Kim}

\author{
Ramona Neferu, Alice Yi \\ Faculty Reviewer: Richard Kim, MD, FRCPC (Division of Clinical Pharmacology)
}

Dr Richard Kim is currently a professor and the chair of the Division of Clinical Pharmacology at Western University and the Director for the Centre for Clinical Investigation and Therapeutics at the London Health Sciences Centre (LHSC). He also holds the Wolfe Medical Research Chair in Pharmacogenomics and is at the forefront of advancing the specialty of clinical pharmacology-a growing field that promises to change the way we deliver healthcare in Canada and abroad. Dr Kim shared his insights into the future of personalized medicine and his roles as a clinician, researcher, administrator, and teacher.

UWOMJ: Tell us a bit about yourself and how you came to be a clinical pharmacologist.

Richard Kim: I grew up in Saskatchewan and graduated in 1987 from medical school at the University of Saskatchewan. I then did a rotating internship and 3 years of internal medicine, both in Saskatoon, where there were good role models in the field of clinical pharmacology. In 1991 I received funding for a 3-year research fellowship at Vanderbilt University in clinical pharmacology. Along with my wife and our 2-year-old, we put everything in our minivan and drove from Saskatoon to Nashville, Tennessee. I didn't know what kind of experience it would be, but looking back, it was one of the best decisions I've made.

My research dealt with interpatient molecular determinants of variation in drug response. We wanted to find out why people vary in their drug exposure response when prescribed the same dose and medication. By 1994 our research was looking pretty promising, and I was offered a faculty position. I stayed as a faculty member there for 12 more years.

By mid-2000 I had a very large program with multimillion-dollar grants and a very large number of trainees, both MDs and PhDs. We had to decide if we wanted to continue to grow in that setting or try something different. I was passionate about translating our research to patient care, so along with 2 faculty members and 3 postdoctoral fellows, we took the chance to come to Western in 2006. I had no idea what would happen leaving a well-established institution, but coming back to Canada and to a city such as London had the potential of making a more immediate difference in patient care.

Here, we worked with the hospitals to create the first personalized medicine clinic. We started doing pre-emptive genetic testing of relevance to drug response as a way of preventing adverse drug reactions. We first focused on warfarin and later expanded to cardiovascular drugs, statins, antiplatelet drugs, anti-inflammatory immunosuppressants, and breast cancer drugs like tamoxifen. In pediatrics, we have had significant ongoing efforts in using pharmacogenomics and personalized medicine. We also hope to further enhance the benefits and safety of mental health medications known to be affected by genetic differences. In early 2016, we will introduce a hospital-wide personalized medicine approach, starting with the oral anticoagulant warfarin, to further demonstrate feasibility of our approach as well as objectively demonstrate cost-effectiveness through reduced length of stay, better discharge planning, reduced readmission rates, and better patient outcomes.

\section{What sorts of challenges do clinical pharmacologists deal with?}

There are variations of responses, gene products, age, gender, diet, complex drug interactions, and genomic differences that must be further integrated into delivery of care. Many patients are very different from the patients in randomized drug trials, so we have to do our best to identify and rescue them from lack of benefit.

We can use technology like genomics and mass spectrometry to measure drug levels and provide better care for our patients. We try to optimize drug dosages for our patients, even if that means administering a nonstandard dose. This is done with full permission and consent from the patients and their families, as well as other physicians and pharmacists involved in the care of such patients. This takes a bit of guts, specialty training, clinical judgment, and ability to document nonstandard dosages that other groups would not use.

Can you talk about the clinical pharmacology program for medical residents as well as for graduate students?

Western now has the largest clinical pharmacology program in the country, and second largest in North America. We look for candidates who have unbridled enthusiasm for translational research and patient care. We have partnered with other subspecialties to offer combined 3-year subspecialty residency programs after 3 core years in internal medicine or pediatrics. We provide training related to pharmacotherapy, pharmacokinetics, and pharmacogenetics while tailoring our program to each resident's intended scope of practice.

Graduate students are also an important part of our personalized medicine program, particularly in terms of innovative technologies and bench-to-bedside research. In addition, interaction with other faculties at Western has been a major benefit; we are already working with the Ivey School of Business on how to scale up our model of personalized medicine to other centers, and are working with the engineering faculty to use smarter technology in data encryption, confidentiality, bioinformatics, and data mining. 
Can you describe a day in your life as a clinical pharmacologist?

During the day I put in a significant amount of effort into our program of personalized medicine-based patient care. We have daily rounding with residents, pharmacists, and other trainees who participate in the care of inpatients who are consulted to us. I also hold 2 weekly half-day personalized medicine clinics. Since starting our personalized medicine clinic at LHSC in 2008, we have looked after over 3000 patients through the clinic and inpatient consultation service.

We see personalized drug therapy as its own speciality that provides outstanding multidisciplinary patient care. For example, even though I hold a half-day clinic at the London Regional Cancer Program, I'm not an oncologist by training. However, we are experts on some of the drugs they use and we are able to provide genomics-guided dosing. Our clinical pharmacology residents see many patients on the wards, and every day we are consulted by different subspecialties for whom we do timely genotyping to provide drug therapy recommendations and follow-up care. We accurately estimate safe and effective drug dosages for various patients, rather than using the traditional iterative approach of slowly increasing the dose from a low baseline dose, which does not work well in conditions where optimized treatment is urgently needed.

At night and on weekends, I spend time writing papers and working on research grants, which extends the 40-50 hour work week closer to 70-80 hours per week for me. All physicians must balance their number of academic and clinical activities. Since there is no other clinic in the country that has a similar model of care, in a way our clinic can be viewed as research. But it is foremost patient-centred care. Creating that model of care is what our team is really doing, and we hope that our approach can be used throughout Canada and beyond.

Every time we help prevent a near death situation using genomics technologies, it is one of the most comforting thoughts for me. We see clear patient benefits, and that is why I have become such an advocate of innovation in health care such as our personalized medicine program that I am so passionate about.

How can we adopt personalized medicine to improve the efficiency in our healthcare system?

Physicians tend to be very cautious in adopting new frameworks, but if we do not leverage new technology and teaching capabilities to provide better care, we run the risk of doing more of the same things faster, which may not be sustainable in terms of health care expenditure.

Since it is actually cheaper to prevent severe toxicities and improper dosing than to treat the adverse drug events when they happen, our approach, focusing on "the right dose of the right drug for the right patient," has great potential to reduce overall healthcare costs. This is especially true for the increasing number of elderly patients who are often admitted into hospitals due to drug-related issues. We can prevent hospitalizations by providing genomics-guided advice through pharmacists or physicians who look after those patients in long term care facilities using on-demand telemedicine capabilities that already exist in Ontario.
How can clinical pharmacology be integrated into the current healthcare system?

For personalized medicine as a patient care model to really take off, we need to better understand what works or does not work in a real-world clinical setting. There is little doubt we have to generate robust and objective data on the cost-benefit trade-off of our approach to gain the support of provinces and regulatory agencies such that all healthcare providers are more able to adopt this new framework. We hope to show that this is an economically sustainable model. Our efforts in personalized medicine-based care have been nearly a decade in the making. Our goal is to do the right thing in a titrated and systematic fashion so that we don't overpromise and underdeliver.

Our team is also thinking about how we can help other centres adopt our model of care, but also help them accelerate instead of redoing what we've done from the beginning. We can provide further guidance and logistics support through teleconferencing and consultations. The good thing is that our approach was recognized in an advisory panel on healthcare innovation led by Dr David Naylor, former president of University of Toronto, for consideration by the federal minister, tasked with what Canada should do to be excellent in healthcare for the next decade. The recommendations urged the government to invest an additional $\$ 1$ billion per year in healthcare focusing on 5 initiatives, 1 of them being personalized medicine and pharmacogenomics approaches that teams like ours have been initiating. That gave us further confidence. We now have the national visibility to say we are being recognized as making a difference.

\section{Can you speak to the future employment prospects in clinical pharmacology?}

Although we can't predict the future, I'm confident that the field of personalized medicine is where the next wave of long-term innovative careers will be heading. We're going to generate the next versions of physicians, and there is a huge potential for doing something that ignites passion and innovation in this field. I don't see why physicians who can use technology and can integrate more complex clinical information of relevance to patient care would be disadvantaged.

For medical students, sometimes training related to personalized medicine doesn't seem as relevant at the moment and may seem too futuristic, but I'm sure people said the same thing about smartphones 10 years ago. 